\title{
The Two-Component Regulatory System VicRK is Important to Virulence of Streptococcus equi Subspecies equi
}

\author{
Mengyao Liu, Michael J. McClure, Hui Zhu, Gang Xie and Benfang Lei* \\ Department of Veterinary Molecular Biology, Montana State University, Bozeman, Montana 59717, USA
}

\begin{abstract}
This study aims at evaluating the importance of the two-component regulatory system VicRK to virulence of the horse pathogen Streptococcus equi subspecies equi and the potential of a vicK mutant as a live vaccine candidate using mouse infection models. The vicK gene was deleted by gene replacement. The $\Delta v i c K$ mutant is attenuated in virulence in both subcutaneous and intranasal infections in mice. $\Delta v i c K$ grows less slowly than the parent strain but retains the ability of $S$. equi to resist to phagocytosis by polymorphoneuclear leukocytes, suggesting that the vicK deletion causes growth defect. $\Delta v i c K$ infection protects mice against reinfection with a wild-type $S$. equi strain. Intranasal $\Delta v i c K$ infection induces production of anti-SeM mucosal IgA and systemic IgG. These results indicate that VicRK is important to $S$. equi growth and virulence and suggest that $\Delta v i c K$ has the potential to be developed as a live $S$. equi vaccine.
\end{abstract}

\section{INTRODUCTION}

Bacterial pathogens produce many two-component regulatory systems to regulate gene expression by specific environmental signals [1]. These systems consist of membrane protein sensors and cognate cytoplasmic response regulators. The regulator is phosphorylated by the sensor in response to a specific signal, activating or repressing the transcription of target genes. The two-component regulatory system VicRK or YycFG is specific for Gram-positive bacteria. The regulator component VicR is essential in Bacillus subtilis [2], Staphylococcus aureus [3], and Streptococcus pneumoniae [4-5] but appears not to be essential in Streptococcus pyogenes [6]. The deletion of the vicK gene can be readily inactivated in S. pneumoniae [7], Streptococcus mutans [8], and $S$. pyogenes [6] but not in B. subtilis [2] and S. aureus [3]. Conditional and unconditional vicRK mutants display various phenotypes, including defects in morphology and cell wall synthesis, decreased competence, sensitivity to antibiotics and fatty acids, defects in biofilm formation, and attenuated virulence, growth defect, and sensitivity to osmotic pressure [3, 6, 8-11].

The vicRK system of Gram-positive bacterium Streptococcus equi subspecies equi (S. equi) has not been studied. This pathogen causes equine strangles, a highly contagious purulent lymphadenitis [12-13]. The infection initially causes nasal discharge and fever and, then, leads to abscess formation in local lymph nodes, causing respiratory difficulty. Although the infection at the lymph nodes cause massive infiltration of polymorphoneuclear leukocytes (PMNs) [14], S. equi resists phagocytosis by PMNs and rapidly multiplies, forming an abscess of large numbers of degenerating PMNs and long chains of S. equi [15]. The hyaluronic acid

*Address correspondence to this author at Department of Veterinary Molecular Biology, Montana State University, P.O. Box 173610, Bozeman, Montana 59717, USA; E-mail: blei@montana.edu capsule and S. equi M-like protein (SeM) are both required for the resistance to phagocytosis by PMNs [16-17]. Most horses recovered from strangles have immunity against $S$. equi reinfection for up to 5 years [18]. It is believed that the immunity is mediated by mucosal antibodies specific to SeM and other protective antigens. An intranasal vaccine made of live attenuated strain has been used in USA, which lacks the hyaluronic acid capsule, and various adverse effects, including pharyngeal lymphadenopathy, limb edema, and bastard strangles abscesses, have been reported [15].

This study aims at evaluating the importance of VicRK to S. equi virulence and the potential of a vicK deletion mutant as a live vaccine using mouse infection models. We found that the vicK deletion attenuated $S$. equi virulence in mouse models of subcutaneous and intranasal infections and that infection with a vicK deletion mutant confers protection against subsequent infection with wild-type $S$. equi and induces production of mucosal and systemic immunoglobins to $\mathrm{SeM}$ in nasal infection.

\section{MATERIALS AND METHODS}

\section{Bacterial Strains and Growth}

S. equi strain SEM1 was isolated in 2003 from a horse with strangles in Montana, USA. SEM1 and its mutant were routinely grown in Todd-Hewitt broth supplemented with $0.2 \%$ yeast extract (THY) in $5 \% \mathrm{CO}_{2}$ at $37^{\circ} \mathrm{C}$ without and with $150 \mathrm{mg} /$ liter spectinomycin, respectively.

\section{Generation of a vicK Deletion Mutant}

A vicK deletion mutant $(\Delta v i c K)$ of S. equi SEM1 was generated by gene replacement (Fig. 1). The upstream and downstream flanking fragments of the deleted internal fragment (bases 451-1282) of vicK were PCR-amplified using paired primers 5'- GAAGCTTCTTATGACTAAGGACATCATTGGAAC-3'/5'-GAGATCTGGTGTAAGGTGAGTC ACTGTC-3' and 5'-AGGATCCCCTTTACCATTGTG TTA CCTTACG-3'/5'-AGTCGACCCTGTATCCGTCAGCATG 
AC-3', respectively. The PCR products of the upstream and downstream fragments were sequentially cloned into pGRV [19] at the HindIII/BglII and BamHI/SalI sites, respectively, to yield pGRV- $\Delta v i c K$. pGRV- $\Delta v i c K$ was introduced into $S$. equi strain SEM1 by electroporation using the conditions described previously for S. pyogenes [6], and the sample was plated on THY agar plate with $150 \mathrm{mg} /$ liter spectinomycin. The obtained colonies, which could be derived from a single or double crossover recombination, were screened by PCR using primers 5'-GAGACTGCTC AAAAGCAGCTC-3' and 5'-GATTTGACTCAATCAAGTAGC-3' and DNA sequencing analyses to identify the desired deletion mutant.

\section{S. equi Growth in Rabbit Blood}

S. equi strains were harvested at the exponential growth phase, washed three times with pyrogen-free Dulbecco's phosphate-buffered saline (DPBS), and inoculated at $2 \times 10^{4}$ $\mathrm{cfu} / \mathrm{ml}$ in heparinized rabbit blood. The samples were rotated end-to-end at $37^{\circ} \mathrm{C}$ for $4 \mathrm{~h}$, and numbers of viable $S$. equi in the samples and actual inocula were determined by plating on THY agar. Growth factor is defined as the ratio of colony-forming units (cfu) of each sample after 4-h incubation to cfu in the inoculum.

\section{Phagocytosis Assay}

Phagocytosis assay was performed as described previously $[6,20]$. Briefly, S. equi SEM1 wild-type and $\Delta v i c K$ cells from exponential growth phase in THY were washed with phosphate-buffered saline (PBS) and labeled with 0.75 $\mu \mathrm{g} / \mathrm{mL}$ FITC in PBS at $37^{\circ} \mathrm{C}$ for $20 \mathrm{~min}$. The labeled bacteria were washed and suspended at $1 \times 10^{9} \mathrm{cfu} / \mathrm{ml}$ in PBS. Ten $\mu l$ of the labeled bacteria were mixed with $100 \mu$ l of nonimmune heparinized rabbit or horse blood and incubated with gentle shaking at $37^{\circ} \mathrm{C}$ for 5 or $15 \mathrm{~min}$. The samples were immediately processed using an Immunolyse Kit (Beckman Coulter) according to the manufacturer's protocol and analyzed by flow cytometry. The percentage of PMNs with fluorescent bacteria was used as a measure of phagocytosis efficiency.

\section{Mouse Infections}

S. equi strains were harvested at exponential phase, washed with DPBS, and inoculated subcutaneously or intranasally at inocula specified in figure legends into groups of 8 female outbred CD-1 Swiss mice. Survival rates were examined daily for 20 days after inoculation. At the end of the intranasal infection experiment, blood was collected from the surviving mice via cardiac puncture, and the nasal wash fluids were then obtained as follows. The trachea was perforated, a small tube was inserted into the opening, and the nasal cavity was slowly flushed with $1.0 \mathrm{ml}$ DPBS through the tube. All animal procedures were approved by the Institutional Animal Care and Use Committee at Montana State University, Bozeman, USA.

\section{Enzyme-linked Immunosorbant Assay (ELISA) and Western Blotting Analysis}

Relative levels of anti-SeM IgG in sera of mice recovered from the intranasal $S$. equi infections were estimated by ELISA and Western blotting using a truncated recombinant
SeM containing amino acids 38 to $260\left(\mathrm{SeM}^{38-260}\right)$ with described procedures [21]. Briefly for ELISA, microtiter plates were coated overnight with $\mathrm{SeM}^{38-260}$ at a concentration of $0.25 \mu \mathrm{g} /$ well. The plates were washed four times with PBS containing $0.1 \%$ (vol/vol) Tween 20 (PBS-T), blocked with $0.1 \%$ bovine albumin (BSA) in PBS-T for $2 \mathrm{~h}$ at room temperature, and washed as described above. The plate was incubated with $100 \mu \mathrm{l} /$ well of mouse sera diluted at 1:100 to $1: 51200$ in $0.1 \%$ BSA in PBS-T and washed as described above. The wells were incubated with goat anti-mouse IgG $(\mathrm{H}+\mathrm{L})$-peroxidase conjugate (1:4,000 dilution). The plates were washed as described above and washed four times with PBS to remove Tween 20. The plates were developed with $100 \mu \mathrm{l} /$ well of ABTS solution for $30 \mathrm{~min}$ and the absorbance was measured at $405 \mathrm{~nm}$. Titers were determined by the geometric method. The presence of $\mathrm{SeM}^{38-260}$-specific IgA in the nasal wash samples were determined by $\mathrm{A}_{405}$ in the ELISA assay as described above using $100 \mu \mathrm{l}$ of 2 -folddiluted nasal wash samples and goat anti-mouse IgA HRP conjugate (Bethyl Laboratories, Inc.).

\section{RESULTS}

\section{S. equi vicK Deletion Mutant}

The $S$. equi vicRK genes were found by blasting the $S$. equi genome database (http://www.sanger.ac.uk/Projects/ $\mathrm{S}$ _equi) with the $S$. pyogenes vicRK sequence. Gene replacement strategy was used to generate vicK-deletion mutant (Fig. 1A). The vector pGRV has two genes aad and $\mathrm{cmR}$ for selections with spectinomycin and chloramphenicol, respectively. The two upstream and downstream flanking fragments of the internal vicK fragment from Tyr151 to Ser427 to be deleted were cloned at the upstream and downstream ends of the aad gene, respectively, resulting in suicide plasmid pGRV- $\Delta v i c K$. Single crossover between one flanking fragment in pGRV- $\Delta v i c K$ and the homologous region in the genome would lead to the insertion of the whole plasmid into $S$. equi genome, resulting in strains resistant to both spectinomycin and chloramphenicol. Double crossover at both of the flanking fragments would lead to the replacement of the vicK internal fragment with the aad gene, resulting in vicK deletion strains with resistance only to spectinomycin. The colonies on spectinomycin agar plates were tested for resistance to chloramphenicol. Three putative $\Delta v i c K$ strains, which were spectinomycin-resistant and chloramphenicol-sensitive, were obtained. PCR analyses using the primers located beyond the deleted fragment resulted in the PCR product from these strains that were expectedly bigger than that from the wild-type strain because the replacing fragment was bigger than the displaced vicK fragment (Fig. 1B). DNA sequencing confirmed the desired deletion. One deletion strain was randomly chosen for further characterization.

\section{Growth of $\Delta v i c K$ in THY and Rabbit Blood}

The growth curve of the $\Delta v i c K$ mutant in THY displays a longer early growth phase and smaller slope in the exponential growth phase than that of the parent strain (Fig. 2A), indicating that the vicK deletion detrimentally affects $S$. equi growth. The effect of the deletion on $S$. equi growth in blood was also examined. The wild-type and $\Delta v i c K S$. equi strains 


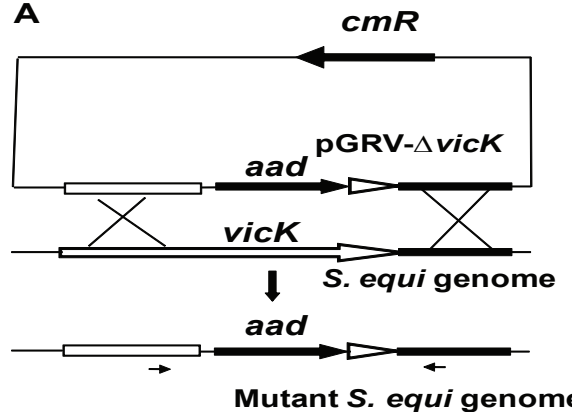

B

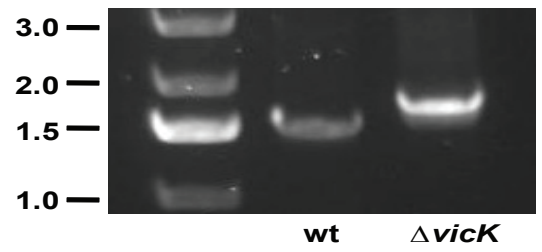

Fig. (1). Deletion of the vicK gene. A) schematic for vicK deletion by gene replacement. The two flanking fragments of the internal vicK fragment to be deleted were cloned into the up- and downstream ends of the aad gene in pGRV. The resulting plasmid pGRV- $\Delta v i c K$ was introduced into $S$. equi, and double crossover in the homologous regions between the plasmid and $S$. equi genome resulted in $\Delta v i c K$ mutants. B) PCR confirmation of the vicK deletion. The picture shows agarose gel analysis of PCR reactions using mutant (lane $\Delta v i c K$ ) or wild-type (lane wt) genomic DNA as template and primers indicated by the arrows under the mutant genome.

were inoculated into $1 \mathrm{ml}$ heparinized rabbit blood at an inoculum of approximately 20,000 cfu. The samples were incubated for $4 \mathrm{~h}$, and the numbers of the bacteria in the samples and inocula at time zero were determined by plating. The growth factors, the ratio of cfu in the sample at $4 \mathrm{~h}$ over cfu at time zero, were 250 and 66 for the wild type and $\Delta v i c K$ strains, respectively (Fig. 2B). Thus, the $\Delta v i c K$ mutant has significantly reduced ability to grow in rabbit blood $(\mathrm{P}<$ 0.0001 ).

\section{No Effect of the vicK Deletion on Resistance of $S$. equi to Phagocytosis by PMNs}

To determine whether the $\Delta v i c K$ deletion affects the resistance of $S$. equi to phagocytosis by PMNs, the phagocytosis of wild-type and $\Delta v i c K$ bacteria by PMNs in non-immune horse and rabbit blood was compared. FITC-labeled wildtype $S$. equi, $\Delta$ vicK mutant, and S. pyogenes spy1718::aad mutant were incubated with heparinized horse or rabbit blood for 5 and $15 \mathrm{~min}$, and percentages of PMNs associated with fluorescent bacteria were quantified using flow cytometry analysis. The spy1718::aad mutant of S. pyogenes, which is no longer resistant to phagocytosis by PMNs, was used as a positive control in the assay. The percentages of PMNs with associated wild-type $S$. equi and spy1718::aad were low and high, respectively, indicating that the assay worked well to evaluate resistance of the bacteria to phagocytosis. There was no significant difference in the percentages of PMNs associated with wild-type and $\Delta v i c K$ bacteria at both time points and in both horse and rabbit blood (Fig. 3), indicating that the $\Delta v i c K$ mutant retains the ability of $S$. equi to resist to phagocytosis by PMNs.
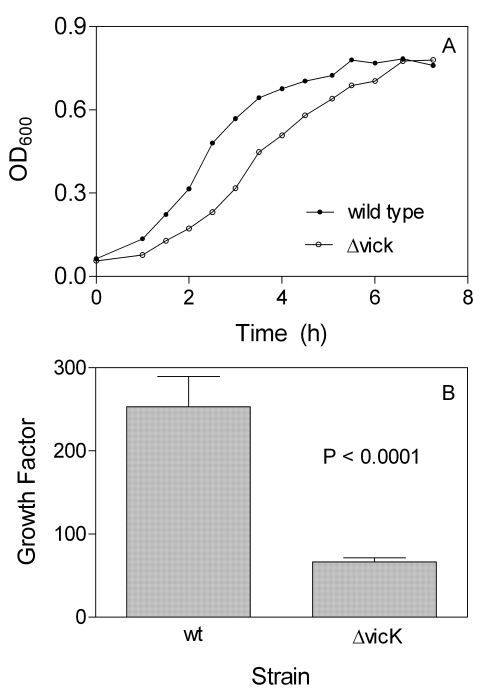

Fig. (2). A) growth curves of wild-type and $\Delta v i c K$ strains in THY. Cultures at the exponential phase were inoculated into fresh THY, and $\mathrm{OD}_{600}$ was measured at the indicated times. B) growth of wildtype and $\Delta v i c K$ strains in rabbit blood. Approximately $2 \times 10^{4} \mathrm{cfu}$ of each strain was inoculated into $1 \mathrm{ml}$ blood in triplicate. Numbers of the bacteria in inocula and in the samples after end-to-end rotation at $37^{\circ} \mathrm{C}$ for $4 \mathrm{~h}$ were determined by plating. The growth factor (cfu at $4 \mathrm{~h} / \mathrm{cfu}$ at $0 \mathrm{~h}$ ) $\pm \mathrm{SD}$ is presented.

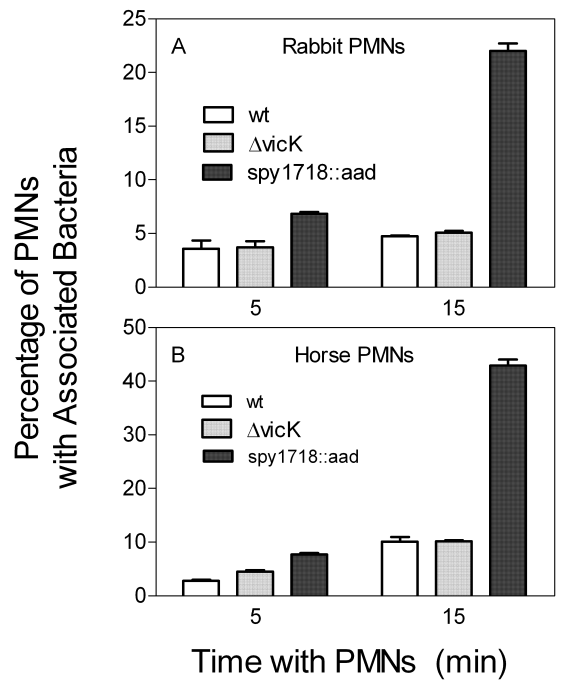

Fig. (3). Association of wild-type and $\Delta v i c K$ bacteria with rabbit (A) and horse (B) PMNs. FITC-labeled bacteria $\left(10^{7} \mathrm{cfu}\right)$ were incubated with $100 \mu \mathrm{l}$ heparinized blood at $37^{\circ} \mathrm{C}$ for 5 or $15 \mathrm{~min}$. Red blood cells were lysed using an Immunolyse kit, and percentages of PMNs with associated (bound and phagocytosed) bacteria determined by flow cytometry are presented. A $S$. pyogenes spy1718::aad mutant was included as a positive control.

\section{Attenuation of $S$. equi Virulence by vicK Deletion}

Group of 8 mice were subcutaneously inoculated with 1.1 x $10^{8}$ cfu wild-type or $\Delta v i c K$ mutant strains. Seven of the 8 mice infected with the wild-type $S$. equi strain died, whereas 7 of the 8 mice inoculated with $\Delta v i c K$ survived (Fig. 4A). The infection was performed in a model of intranasal infec- 
tion as well. All the 8 mice infected with $\Delta v i c K$ survived, whereas 5 of the 8 mice infected with the wild-type $S$. equi strain died (Fig. 4B). These results indicate that the vicK deletion significantly attenuated $S$. equi virulence in both mouse models of subcutaneous $(\mathrm{P}=0.0066)$ and nasal $(\mathrm{P}=$ 0.0085) infections.

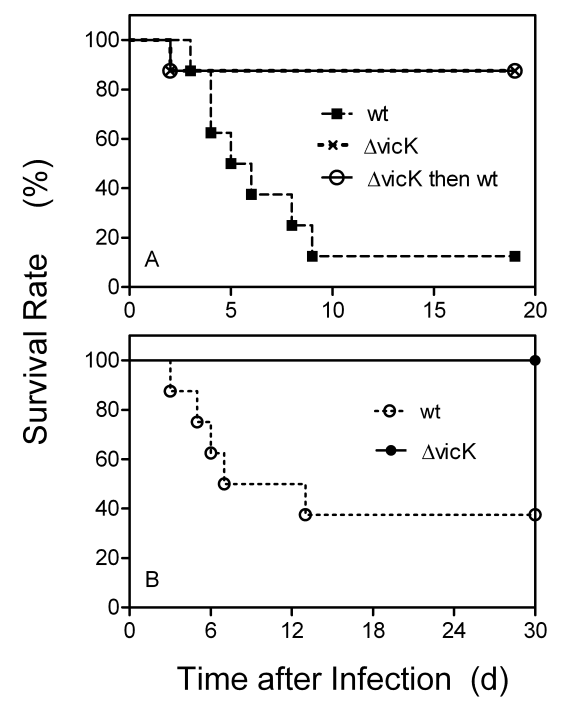

Fig. (4). Attenuation of $S$. equi virulence by vicK deletion and protection of mice against wild-type $S$. equi infection conferred by preceding $\Delta v i c K$ infection. Groups of $8 \mathrm{CD}-1$ mice were inoculated subcutaneously (A) and intranasally (B) with $1.1 \times 10^{8} \mathrm{cfu}$ of each strain, and survival rates were determined daily. Panel A also includes the results of the subcutaneous infection with $1.5 \times 10^{8} \mathrm{cfu}$ of the wild-type strain on the 7 mice survived from the subcutaneous $\Delta v i c K$ infection (the open circles).

\section{$\Delta v i c K$ Infection Confers Protection of Mice against Rein- fection with Wild-Type $S$. equi}

To test whether $\Delta v i c K$ infection confers immunity against $S$. equi infection, the seven mice recovered from the subcutaneous $\Delta v i c K$ infection was reinfected subcutaneously with $1.5 \times 10^{8}$ cfu wild-type $S$. equi 30 days after the first infection and monitored for 18 days. Six of the 7 mice survived the reinfection (Fig. 4A), suggesting that the $\Delta v i c K$ infection induces immunity against $S$. equi infection.

\section{Intranasal $\Delta v i c K$ Infection Induces SeM-Specific Muco- sal IgA and Systemic IgG}

To examine the humoral immune responses in the intranasal $\Delta v i c K$ infection, nasal wash and serum samples were collected from the 8 mice infected intranasally with $\Delta v i c K$ and 3 surviving mice infected with the wild-type $S$. equi 30 days after infection. Half of the nasal wash samples from the mice infected with $\Delta v i c K$ had similar levels of $\mathrm{SeM}^{38-260}-\operatorname{IgA}$ reactivity with those from the mice infected with the wildtype strains. Similarly, these 4 mice with higher $\operatorname{Ig}$ A levels also had higher levels of SeM-specific systemic IgG (Fig. 5B). Western blotting analysis was used to confirm the presence of SeM-specific IgG. The wild-type sera and 5 of the 8 $\Delta v i c K$ samples had strong immunoreactions with $\mathrm{SeM}^{38-260}$ in Western blotting analysis (Fig. 5C). Thus, the $\Delta v i c K \mathrm{mu}-$ tant has the ability to induce mucosal and systemic immune responses, though there was host variation in these responses caused by $\Delta v i c K$ infection.

A B
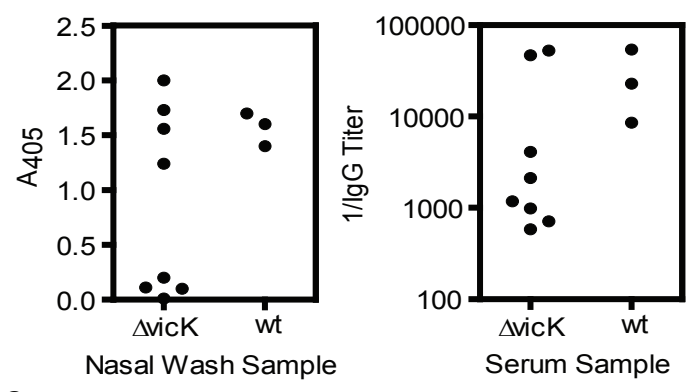

C

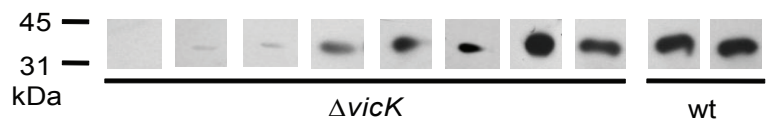

Fig. (5). Assessment of SeM-specific mucosal IgA and systemic IgG production in mice with the intranasal $S$. equi infection by ELISA and Western immunoblot. Nasal wash and serum samples were collected from the surviving mice in the nasal infections with wild-type and $\Delta v i c K$ strains of Fig. 4B on day 30 after inoculation. A) immunoreactivity of $\mathrm{SeM}^{38-260}$ with $\operatorname{IgA}$ in the nasal wash samples. B) the reciprocal of anti-SeM ${ }^{38-260} \operatorname{IgG}$ titers in the serum samples. C) immunoblots demonstrating anti-SeM ${ }^{38-260} \mathrm{IgG}$ in the serum samples, which were diluted by 1:1000 in the assay.

\section{DISCUSSION}

VicK is essential in B. subtilis [2] and S. aureus [3] but not in S. pneumoniae [7], S. mutans [8], and S. pyogenes [6]. We successfully deleted the vicK gene of $S$. equi. Thus, VicK is not essential in S. equi. However, the $\Delta v i c K$ mutant is attenuated in virulence in both mouse models of subcutaneous and intranasal $S$. equi infections, indicating that VicRK is important to virulence. The results provide the further evidence for the importance of VicRK to virulence of Gram-positive pathogens.

S. equi $\Delta v i c K$ mutant does not grow as well as the wildtype strain in both THY and blood, suggesting that the vicK deletion causes defect in growth, a plausible reason that likely contributes to the attenuation of $S$. equi virulence in the mouse infection models. This suggestion is further supported by the observations that both the wild-type and $\Delta v i c K$ mutant strains are resistant to phagocytosis by PMNs, which suggest that VicRK is not required for the evasion of $S$. equi to the innate immunity. As introduced earlier, the various phenotypes have been described for the vicRK mutants of the various pathogens. Whether the phenotypes are specific to particular organisms is not known. The phenotypes of the $S$. equi $\Delta$ vicK mutant are similar to those of the $S$. pyogenes $\Delta v i c K$ mutant [6], suggesting that the growth defect phenotype may be a common feature of the vicRK mutants of various Gram-positive pathogens.

The $\Delta v i c K$ mutant appears to possess the properties of a potential live vaccine. First, it is attenuated in virulence in the mouse infection models. Secondly, $\Delta v i c K$ inoculation protects mice against subsequent infection with wild-type $S$. equi. Thirdly, most of the mice with intranasal $\Delta v i c K$ infection produce mucosal IgA and systemic IgG specific to protective antigen SeM. However, whether $\Delta v i c K$ can be an 
effective live vaccine and whether the $\Delta v i c K$ mutant has any advantages over the current live $S$. equi vaccine require the test of the mutant in horses since $S$. equi does not naturally infect mice. We hope to perform this expensive test in future when funds are available.

\section{ACKNOWLEDGEMENTS}

This work was supported by grants from the NRI Competitive Grants Program of the U.S. Department of Agriculture (grant no. 2006-01690 and 2007-35204-18306) and P20 RR-020185 from the National Center for Research Resources, and the Montana State University Agricultural Experimental Station and USDA Formula Funds.

\section{REFERENCES}

[1] Hoch JA. Two-component and phosphorelay signal transduction. Curr Opin Microbiol 2000; 3(2): 165-70.

[2] Fabret C, Hoch JA. A two-component signal transduction system essential for growth of Bacillus subtilis: Implications for antiinfective therapy. J Bacteriol 1998; 180(23): 6375-83.

[3] Martin PK, Li T, Sun D, Biek DP, Schmid MB. Role in cell permeability of an essential two-component system in Staphylococcus aureus. J Bacteriol 1999; 181(12): 3666-73.

[4] Lange R, Wagner C, de Saizieu A, et al. Domain organization and molecular characterization of 13 two-component systems identified by genome sequencing of Streptococcus pneumoniae. Gene 1999; 237(1): 223-34.

[5] Throup JP, Koretke KK, Bryant AP, et al. A genomic analysis of two-component signal transduction in Streptococcus pneumoniae. Mol Microbiol 2000; 35(3): 566-76.

[6] Liu M, Hanks TS, Zhang J, et al. Defects in ex vivo and in vivo growth and sensitivity to osmotic stress of group A Streptococcus caused by interruption of response regulator gene vicR. Microbiology 2006; 152(Pt 4): 967-78.

[7] Kadioglu A, Echenique J, Manco S, Trombe MC, Andrew PW. The MicAB two-component signaling system is involved in virulence of Streptococcus pneumoniae. Infect Immun 2003; 71(11): 6676-9.
[8] Senadheera MD, Guggenheim B, Spatafora GA, et al. A VicRK signal transduction system in Streptococcus mutans affects gtfBCD, $g b p B$, and $f t f$ expression, biofilm formation, and genetic competence development. J Bacteriol 2005; 187(12): 4064-76.

[9] $\mathrm{Ng}$ WL, Kazmierczak KM, Winkler ME. Defective cell wall synthesis in Streptococcus pneumoniae R6 depleted for the essential PcsB putative murein hydrolase or the $\mathrm{VicR}(\mathrm{YycF})$ response regulator. Mol Microbiol 2004; 53(4): 1161-75.

[10] Echenique JR, Trombe MC. Competence repression under oxygen limitation through the two-component MicAB signal-transducing system in Streptococcus pneumoniae and involvement of the PAS domain of MicB. J Bacteriol 2001; 183(15): 4599-608.

[11] Mohedano ML, Overweg K, de la Fuente A, et al. Evidence that the essential response regulator $\mathrm{YycF}$ in Streptococcus pneumoniae modulates expression of fatty acid biosynthesis genes and alters membrane composition. J Bacteriol 2005; 187(7): 2357-67.

[12] Timoney JF. Strangles. Vet Clin North Am Equine Pract 1993; 9(2): 365-74

[13] Harrington DJ, Sutcliffe IC, Chanter N. The molecular basis of Streptococcus equi infection and disease. Microbes Infect 2002; 4(4): 501-10.

[14] Mukhtar MM, Timoney JF. Chemotactic response of equine polymorphonucelar leucocytes to Streptococcus equi. Res Vet Sci 1988; 45(3-4): 225-9.

[15] Timoney JF. The pathogenic equine streptococci. Vet Res 2004; 35(4): 397-409.

[16] Timoney JF, Artiushin SC, Boschwitz JS. Comparison of the sequences and functions of Streptococcus equi M-like proteins SeM and SzPSe. Infect Immun 1997; 65(9): 3600-5.

[17] Anzai T, Timoney JF, Kuwamoto Y, Fujita Y, Wada R, Inoue T. In vivo pathogenicity and resistance to phagocytosis of Streptococcus equi strains with different levels of capsule expression. Vet Microbiol 1999; 67 (4): 277-86.

[18] Woolcock JB. Immunity to $S$. equi. Aust Vet J 1975; 51(12): 554-9.

[19] Hanks TS, Liu M, McClure MJ, Lei B. ABC transporter FtsABCD of Streptococcus pyogenes mediates uptake of ferric ferrichrome. BMC Microbiol 2005; 5:62.

[20] White-Owen C, Alexander JW, Sramkoski RM, Babcock GF. Rapid whole-blood microassay using flow cytometry for measuring neutrophil phagocytosis. J Clin Microbiol 1992; 30(8): 2071-6.

[21] Lei B, Liu M, Chesney GL, Musser JM. Identification of new candidate vaccine antigens made by Streptococcus pyogenes: Purification and characterization of 16 putative extracellular lipoproteins. J Infect Dis 2004; 189(1): 79-89. 